\title{
The impact of spatial correlation on the statistical properties of the capacity of nakagami-m channels with MRC and EGC
}

\author{
Gulzaib Rafiq $^{{ }^{*}}$, Valeri Kontorovich ${ }^{2}$ and Matthias Pätzold
}

\begin{abstract}
In this article, we have studied the statistical properties of the instantaneous channel capacity ${ }^{\mathrm{a}}$ of spatially correlated Nakagami-m channels for two different diversity combining methods, namely maximal ratio combining (MRC) and equal gain combining (EGC). Specifically, using the statistical properties of the instantaneous signal-tonoise ratio, we have derived the analytical expressions for the probability density function (PDF), cumulative distribution function (CDF), level-crossing rate (LCR), and average duration of fades (ADF) of the instantaneous channel capacity. The obtained results are studied for different values of the number of diversity branches and for different values of the receiver antennas separation controlling the spatial correlation in the diversity branches. It is observed that an increase in the spatial correlation in the diversity branches of an MRC system increases the variance as well as the LCR of the instantaneous channel capacity, while the ADF of the channel capacity decreases. On the other hand, when EGC is employed, an increase in the spatial correlation decreases the mean channel capacity, while the ADF of the instantaneous channel capacity increases. The presented results are very helpful to optimize the design of the receiver of wireless communication systems that employ spatial diversity combining techniques. Moreover, provided that the feedback channel is available, the transmitter can make use of the information regarding the statistics of the instantaneous channel capacity by choosing the right modulation, coding, transmission rate, and power to achieve the capacity of the wireless channel ${ }^{b}$.
\end{abstract}

\section{Introduction}

The performance of mobile communication systems is greatly affected by the multipath fading phenomenon. In order to mitigate the effects of fading, spatial diversity combining is widely accepted to be an effective method $[1,2]$. In spatial diversity combining, such as MRC and EGC, the received signals in different diversity branches are combined in such a way that results in an increased overall received SNR [1]. Hence, the system throughput increases, and therefore, the performance of the mobile communication system improves. It is commonly assumed that the received signals in diversity branches are uncorrelated. This assumption is acceptable if the receiver antennas separation is far more than the carrier wavelength of the received signal [3]. However, due to the scarcity of space on small mobile devices, this

\footnotetext{
* Correspondence: gulzaib.rafiq@uia.no

${ }^{1}$ Faculty of Engineering and Science, University of Agder, P.O.Box 509, NO4898 Grimstad, Norway

Full list of author information is available at the end of the article
}

requirement cannot always be fulfilled. Thus, due to the spatial geometry of the receiver antenna array, the receiver antennas are spatially correlated. It is widely reported in the literature that the spatial correlation has a significant influence on the performance of mobile communication systems employing diversity combining techniques (see, e.g., [4-6], and the references therein).

There exists a large number of statistical models for describing the statistics of the received radio signal. Among these channel models, the Rayleigh [7], Rice [8] and lognormal $[9,10]$ models are of prime importance due to which they have been thoroughly investigated in the literature. Numerous papers have been published so far dealing with the performance and the capacity analysis of wireless communication systems employing diversity combining techniques in Rayleigh and Rice channels (e.g., $[6,11,12])$. However, in recent years the Nakagami$m$ channel model [13] has gained considerable attention due to its good fitness to experimental data and mathematically tractable form $[14,15]$. Moreover, the 
Nakagami- $m$ channel model can be used to study scenarios where the fading is more (or less) severe than the Rayleigh fading. The generality of this model can also be observed from the fact that it inherently incorporates the Rayleigh and one-sided Gaussian models as special cases. For Nakagami- $m$ channels, results pertaining to the statistical analysis of the signal envelope at the combiner output in a diversity combining system, assuming spatially uncorrelated diversity branches, can be found in [16]. For such systems, statistical analysis of the instantaneous channel capacity has also been presented in [17]. Moreover, when using EGC, the system performance analysis is reported in [18]. In addition, a large number of articles can also be found in the literature that study Nakagami- $m$ channels in systems with spatially correlated diversity branches [5,19-24]. Furthermore, the instantaneous capacity of spatially correlated Nakagami- $m$ multiple-input multiple-output (MIMO) channels has also been investigated in [25]. However, to the best of the authors' knowledge, there is still a gap of information regarding the statistical analysis of the instantaneous capacity of spatially correlated Nakagami$m$ channels with MRC and EGC. Specifically, secondorder statistical properties, such as the LCR and the ADF, of the instantaneous capacity of spatially correlated Nakagami- $m$ channels with MRC or EGC have not been investigated in the literature. The aim of this paper is to fill this gap in information.

This paper presents the derivation and analysis of the PDF, CDF, LCR, and ADF of the instantaneous channel capacity $^{c}$ of spatially correlated Nakagami- $m$ channels, for both MRC and EGC. The PDF of the channel capacity is helpful to study the mean channel capacity (or the ergodic capacity) [26], while the CDF of the channel capacity is useful for the derivation and analysis of the outage capacity [26]. The mean channel capacity and the outage capacity are very widely explored by the researchers due to their importance for the system design and performance analysis. The ergodic capacity provides the information regarding the average data rate offered by a wireless link (where the average is taken over all the realizations of the channel capacity) $[27,28]$. On the other hand, the outage capacity quantifies the capacity (or the data rate) that is guaranteed with a certain level of reliability $[27,28]$. However, these two aforementioned statistical measures do not provide insight into the temporal behavior of the channel capacity. For example, the outage capacity is a measure of the probability of a specific percentage of capacity outage, but it does not give any information regarding the spread of the outage intervals or the rate at which these outage durations occur over the time scale. Whereas, the information regarding the temporal behavior of the channel capacity is very useful for the improvement of the system performance [29].
The temporal behavior of the channel capacity can be investigated with the help of the LCR and ADF of the channel capacity. The LCR of the channel capacity is a measure of the expected number of up-crossings (or down-crossings) of the channel capacity through a certain threshold level in a time interval of one second. While, the ADF of the channel capacity describes the average duration of the time over which the channel capacity is below a given level [30,31]. A decrease in the channel capacity below a certain desired level results in a capacity outage, which in turn causes burst errors. In the past, the level-crossing and outage duration analysis have been carried out merely for the received signal envelope to study handoff algorithms in cellular networks as well as to design channel coding schemes to minimize burst errors [32,33]. However, for systems employing multiple antennas, the authors in [29] have proposed to choose the channel capacity as a more pragmatic performance merit than the received signal envelope. Therein, the significance of studies pertaining to the analysis of the LCR of the channel capacity can easily be witnessed for the cross-layer optimization of overall network performance. In a similar fashion, for multi-antenna systems, the importance of investigating the ADF of the channel capacity for the burst error analysis can be argued. It is here noteworthy that the LCR and ADF of the channel capacity are the important statistical quantities that describe the dynamic nature of the channel capacity. Hence, studies pertaining to unveil the dynamics of the channel capacity are cardinal to meet the data rate requirements of future mobile communication systems.

We have analyzed the statistical properties of the channel capacity for different values of the number of diversity branches $L$ and for different values of the receiver antennas separation $\delta_{R}$ controlling the spatial correlation in diversity branches. For comparison purposes, we have also included the results for the mean and variance of the capacity of spatially correlated Rayleigh channels with MRC and EGC (which arise for the case when the Nakagami parameter $m=1$ ). It is observed that for both MRC and EGC, an increase in the number of diversity branches $L$ increases the mean channel capacity, while the variance and the ADF of the channel capacity decrease. Moreover, an increase in the severity of fading results in a decrease in the mean channel capacity; however, the variance and ADF of the channel capacity increase. It is also observed that at lower levels, the LCR is higher for channels with smaller values of the number of diversity branches $L$ or higher severity levels of fading than for channels with larger values of $L$ or lower severity levels of fading. We have also studied the influence of spatial correlation in the diversity branches on the statistical properties of the channel 
capacity. Results show that an increase in the spatial correlation in diversity branches of an MRC system increases the variance as well as the LCR of the channel capacity, while the ADF of the channel capacity decreases. On the other hand, for the case of EGC, an increase in the spatial correlation decreases the mean channel capacity, whereas the ADF of the channel capacity increases. Moreover, this effect increases the LCR of the channel capacity at lower levels. We have confirmed the correctness of the theoretical results by simulations, whereby a very good fitting is observed.

The rest of the paper is organized as follows. Section 2 gives a brief overview of the MRC and EGC schemes in Nakagami- $m$ channels with spatially correlated diversity branches. In Section 3, we present the statistical properties of the capacity of Nakagami- $m$ channels with MRC and EGC. Section 4 deals with the analysis and illustration of the theoretical as well as the simulation results. Finally, the conclusions are drawn in Section 5.

\section{Spatial diversity combining in correlated Nakagami-m channels}

We consider the $L$-branch spatial diversity combining system shown in Figure 1, in which it is assumed that the received signals $x_{l}(t)(l=1,2, \ldots, L)$ at the combiner input experience flat fading in all branches. The transmitted signal is represented by $s(t)$, while the total transmitted power per symbol is denoted by $P_{s}$. The complex random channel gain of the $l$ th diversity branch is denoted by $\hat{h}_{l}(t)$ and $n_{l}(t)$ designates the corresponding additive white Gaussian noise (AWGN) component with variance $N_{0}$. The relationship between the transmitted signal $s(t)$ and the received signals $x_{l}(t)$ at the combiner input can be expressed as

$$
\mathbf{x}(t)=\hat{\mathbf{h}}(t) s(t)+\mathbf{n}(t)
$$

where $\mathbf{x}(t), \hat{\mathbf{h}}(t)$, and $\mathbf{n}(t)$ are $L \times 1$ vectors with entries corresponding to the $l$ th $(l=1,2, \ldots, L)$ diversity branch denoted by $x_{l}(t), \hat{h}_{l}(t)$, and $n_{l}(t)$, respectively. The spatial correlation between the diversity branches arises due to the spatial correlation between closely located receiver antennas in the antenna array. The correlation matrix $\mathbf{R}$, describing the correlation between diversity branches, is given by $\mathbf{R}=E\left[\hat{\mathbf{h}}(t) \hat{\mathbf{h}}^{H}(t)\right]$, where $(\cdot)^{H}$ represents the Hermitian operator. Using the Kronecker model, the channel vector $\hat{\mathbf{h}}(t)$ can be expressed as $\hat{\mathbf{h}}(t)=\mathbf{R}^{\frac{1}{2}} \mathbf{h}(t)$ [34]. Here, the entries of the $L \times 1$ vector $\mathbf{h}(t)$ are mutually uncorrelated with amplitudes and phases given by $\left|h_{l}(t)\right|$ and $\varphi_{l}$, respectively. We have assumed that the phases $\varphi_{l}(l=1,2, \ldots, L)$ are uniformly distributed over $(0,2 \pi]$, while the envelopes $\zeta_{l}(t)=\mid h_{l}$ $(t) \mid(l=1,2, \ldots, L)$ follow the Nakagami- $m$ distribution $p_{\zeta_{1}}(z)$ given by [13]

$$
p_{\zeta \iota}(z)=\frac{2 m_{l}^{m_{l}} z^{2 m_{l}-1}}{\Gamma\left(m_{l}\right) \Omega_{l}^{m_{l}}} \mathrm{e}^{-\frac{m_{l} z^{2}}{\Omega_{l}}}, \quad z \geq 0
$$

where $\Omega_{l}=E\left\{\zeta_{l}^{2}(t)\right\}, \quad m_{l}=\Omega_{l}^{2} / \operatorname{Var}\left\{\zeta_{l}^{2}(t)\right\}$, and $\Gamma(\cdot)$ represents the gamma function [35]. Here, $E\{\cdot\}$ and Var $\{$.$\} denote the mean (or the statistical expectation) and$ variance operators, respectively. The parameter $m_{l}$ controls the severity of the fading. Increasing the value of $m_{l}$ decreases the severity of fading associated with the $l$ th branch and vice versa.

The eigenvalue decomposition of the correlation matrix $\mathbf{R}$ can be expressed as $\mathbf{R}=\mathbf{U} \mathbf{\Lambda} \mathbf{U}^{H}$. Here, $\mathbf{U}$ consists of the eigenbasis vectors at the receiver and the diagonal matrix $\Lambda$ comprise the eigenvalues $\lambda_{l}(l=1,2$, ..., $L$ ) of the correlation matrix $\mathbf{R}$. The receiver antenna correlations $\rho_{p, q}(p, q=1,2, \ldots, L)$ under isotropic scattering conditions can be expressed as $\rho_{p, q}=J_{0}\left(b_{p q}\right)$ [36], where $J_{0}(\cdot)$ is the Bessel function of the first kind of order zero [35] and $b_{p q}=2 \pi \delta_{p q} / \lambda$. Here, $\lambda$ is the wavelength of the transmitted signal, whereas $\delta_{p q}$ represents the spacing between the $p$ th and $q$ th receiver antennas. In this article, we have considered a uniform linear array with adjacent receiver antennas separation represented by $\delta_{R}$. Increasing the value of $\delta_{R}$ decreases the spatial correlation between the diversity branches and vice versa. It is worth mentioning here that the analysis

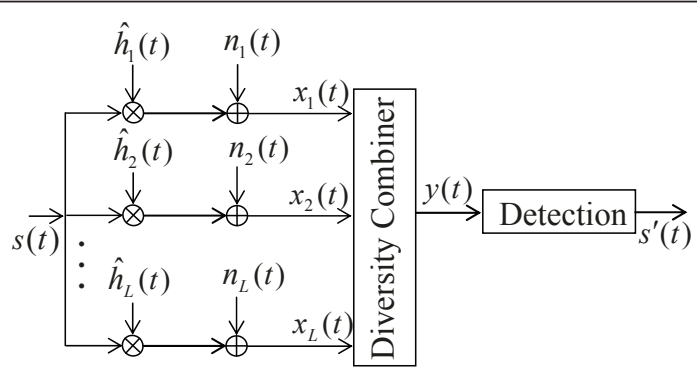

Figure 1 The block diagram representation of a diversity combining system. 
presented in this article is not restricted to any specific receiver antenna correlation model, such as given by $J_{0}$ $(\cdot)$, for the description of the correlation matrix $\mathbf{R}$. Therefore, any receiver antenna correlation model can be used as long as the resulting correlation matrix $\mathbf{R}$ has the eigenvalues $\lambda_{l}(l=1,2, \ldots, L)$.

\subsection{Spatially correlated Nakagami-m channels with MRC}

In MRC, the combiner computes $\mathbf{y}(t)=\hat{\mathbf{h}}^{H}(t) \mathbf{x}(t)$, and hence, the instantaneous SNR $\gamma(t)$ at the combiner output in an MRC diversity system with correlated diversity branches can be expressed as $[1,22]$

$$
\gamma(t)=\frac{P_{s}}{N_{0}} \widehat{\mathbf{h}}^{H}(t) \widehat{\mathbf{h}}(t)=\frac{P_{s}}{N_{0}} \sum_{l=1}^{L} \lambda_{l} \zeta_{l}^{2}(t)=\gamma_{s} \Xi(t)
$$

where $\gamma_{s}=P_{s} / N_{0}$ can be termed as the average SNR of each branch, $\Xi(t)=\sum_{l=1}^{L} \zeta_{l}^{2}(t)$, and $\zeta_{l}(t)=\sqrt{\lambda_{l}} \zeta_{l}(t)$. It is worth mentioning that although we have employed the Kronecker model, the study in [22] reports that (3) holds for any arbitrary correlation model, as long as the correlation matrix $\mathbf{R}$ is non-negative definite. It is also shown in [22] that despite the diversity branches are spatially correlated, the instantaneous SNR $\gamma(t)$ at the combiner output of an MRC system can be expressed as a sum of weighted statistically independent gamma variates $\zeta_{l}^{2}(t)$, as given in (3). The PDF $p_{\xi_{l}^{2}}(z)$ of processes $\zeta_{l}^{2}(t)$ follows the gamma distribution with parameters $\alpha_{l}$ $=m_{l}$ and $\dot{\beta}_{l}=\lambda_{l} \Omega_{l} / m_{l}$ [[37], Equation 1]. Therefore, the process $\Xi(t)$ can be considered as a sum of weighted independent gamma variates. As a result, the PDF $p_{\Xi}(z)$ of the process $\Xi(t)$ can be expressed using [[37], Equation 2] as

$$
p_{\Xi}(z)=\prod_{l=1}^{L}\left(\frac{\dot{\beta}_{1}}{\dot{\beta}_{l}}\right)^{\alpha_{l}} \sum_{k=0}^{\infty} \frac{\varepsilon_{k} z^{\sum_{l=1}^{L} \alpha_{l}+k-1} \mathrm{e}^{-z / \dot{\beta}_{1}}}{\hat{\beta}_{1}^{\sum_{l=1}^{L} \alpha_{l}+k} \Gamma\left(\sum_{l=1}^{L} \alpha_{l}+k\right)},
$$

where

$$
\varepsilon_{k+1}=\frac{1}{k+1} \sum_{i=1}^{k+1}\left[\sum_{l=1}^{L} \alpha_{l}\left(1-\frac{\dot{\beta}_{1}}{\dot{\beta}_{l}}\right)^{l}\right] \varepsilon_{k=0,1,2 \ldots} \varepsilon_{k+1-l,}
$$

$\varepsilon_{0}=1$, and $\dot{\beta}_{1}=\min _{l}\left\{\dot{\beta}_{l}\right\}(l=1,2, \ldots, L)$.

When using MRC, if the diversity branches are uncorrelated having identical Nakagami- $m$ parameters (i.e., when in (3) $\lambda_{l}=1(l=1,2, \ldots, L), \alpha_{1}=\alpha_{2}=\ldots=\alpha_{L}=$ $\alpha$, and $\hat{\beta}_{1}=\hat{\beta}_{2}=\cdots=\dot{\beta}_{L}=\beta$ ), it is shown in [16] that the joint PDF $p_{\Xi \dot{\Xi}}(z, \dot{z})$ of $\Xi(t)$ and its time derivative
$\dot{\Xi}(t)$ at the same time $t$, under the assumption of isotropic scattering, can be written with the help of the result reported in [[16], Equation 35] as

$$
p_{\Xi \dot{\Xi}}(z, \dot{z})=p_{\Xi}(z) \frac{1}{\sqrt{2 \pi \sigma_{\dot{\Xi}}^{2}}} e^{-\frac{\dot{z}^{2}}{2 \sigma_{\dot{\Xi}}^{2}}}, \quad z \geq 0,|\dot{z}|<\infty
$$

where $\sigma_{\dot{\Xi}}^{2}=4 \beta_{x} z\left(\pi f_{\max }\right)^{2}, f_{\max }$ is the maximum Doppler frequency, and $\beta_{x}$ can be expressed as a ratio of the variance and the mean of the sum process $\Xi(t)$, i.e., $\beta_{x}=$ Var $\{\Xi(t)\} / E\{\Xi(t)\}$. Therefore, for uncorrelated diversity branches with identical parameters $\{\alpha=m, \beta=\Omega / m\}, \beta_{x}=$ $\beta$. On the other hand, when the diversity branches are spatially correlated, $\lambda_{l} \neq 1(l=1,2, \ldots, L)$ as well as the eigenvalues are all distinct. Moreover, we have also considered that the parameters $\left\{m_{l}, \Omega_{l}\right\}$ (and therefore $\left\{\alpha_{l}, \hat{\beta}_{l}\right\}$ ) are non-identical. However, as given by (3), even when the diversity branches are spatially correlated and have nonidentical parameters, the process $\Xi(t)$ is still expressed using a sum of statistically independent gamma variates, similar to the uncorrelated scenario considered in [16] to obtain (6). Hence, in our case, we follow a similar approach as in [16], i.e., by assuming that (6) is also valid for the process $\Xi(t)=\sum_{l=1}^{L} \zeta_{l}^{2}(t)$ with parameters $\left.\left\{\alpha_{l}, \hat{\beta}_{l}\right\}\right)$ and finding appropriate value of $\sigma_{\dot{\Xi}}^{2}$. The results show that (6) holds for the process $\Xi(t)$ if the parameter $\beta_{x}$ in $\sigma_{\Xi}^{2}$ is chosen according to $\beta_{x}=\sum_{l=1}^{L}\left(\alpha_{l} \hat{\beta}_{l}^{2}\right) / \sum_{l=1}^{L}\left(\alpha_{l} \hat{\beta}_{l}\right)$. In Section 3 , we will use the results presented in (4) and (6) to obtain the statistical properties of the capacity of Nakagami- $m$ channels with MRC.

\subsection{Spatially correlated Nakagami- $m$ channels with EGC} In EGC, the combiner computes $\mathbf{y}(t)=\varphi^{H} \mathbf{x}(t)$ [4], where $\varphi=\left[\varphi_{1} \varphi_{2}, \ldots, \varphi_{L}\right]^{\mathrm{T}}$ and $(\cdot)^{\mathrm{T}}$ denotes the vector transpose operator. Therefore, the instantaneous SNR $\gamma(t)$ at the combiner output in an $L$-branch EGC diversity system with correlated diversity branches can be expressed as $[1,4,38]$

$$
\gamma(t)=\frac{P_{s}}{L N_{0}}\left(\sum_{l=1}^{L} \sqrt{\lambda_{l}} \zeta_{l}(t)\right)^{2}=\frac{\gamma_{s}}{L} \Psi(t)
$$

where $\Psi(t)=\left(\sum_{l=1}^{L} \zeta_{l}(t)\right)^{2}$, while here the processes $\zeta_{l}(t)$ follow the Nakagami- $m$ distribution with parameters $m_{l}$ and $\dot{\Omega}_{l}=\lambda_{l} \Omega_{l}$. Again we proceed by first finding the PDF $p_{\Psi}(z)$ of the process $\Psi(t)$ as well as the joint PGF $p_{\Psi \dot{\Psi}}(z, \dot{z})$ of the process $\Psi(t)$ and its time derivative $\dot{\Psi}(t)$. However, the exact solution for the PDF of a 
sum of Nakagami- $m$ processes $\sum_{l=1}^{L} \dot{\zeta}_{l}(t)$ cannot be obtained. One of the solutions to this problem is to use an appropriate approximation to the sum $\sum_{l=1}^{L} \zeta_{l}(t)$ to find the PDF $p_{\Psi}(z)$ (see, e.g., [13] and [39]). In this article, we have approximated the sum of Nakagami- $m$ processes $\sum_{l=1}^{L} \zeta_{l}(t)$ by another Nakagami- $m$ process $S(t)$ with parameters $m_{S}$ and $\Omega_{S}$, as suggested in [39]. Hence, the PDF $p_{S}(z)$ of $S(t)$ can be obtained by replacing $m_{l}$ and $\Omega_{l}$ in (2) by $m_{S}$ and $\Omega_{S}$, respectively, where $\Omega_{S}=E\left\{S^{2}(t)\right\}$ and $m_{S}=\Omega_{S}^{2} /\left(E\left\{S^{4}(t)\right\}-\Omega_{S}^{2}\right)$. The $n$ thorder moment $E\left\{S^{n}(t)\right\}$ can be calculated using [39]

$$
\begin{aligned}
E\left\{S^{n}(t)\right\}= & \sum_{n_{1}=0}^{n} \sum_{n_{2}=0}^{n_{1}} \ldots \sum_{n_{L-1}=0}^{n_{L-2}}\left(\begin{array}{c}
n \\
n_{1}
\end{array}\right)\left(\begin{array}{c}
n_{1} \\
n_{2}
\end{array}\right) \ldots\left(\begin{array}{c}
n_{L-2} \\
n_{L-1}
\end{array}\right) \\
& \times E\left\{\dot{\zeta}_{1}^{n-n_{1}}(t)\right\} E\left\{\dot{\zeta}_{2}^{n_{1}-n_{2}}(t)\right\} \ldots E\left\{\dot{\zeta}_{L}^{n_{L-1}}(t)\right\}
\end{aligned}
$$

where $\left(\begin{array}{l}n_{i} \\ n_{j}\end{array}\right)$, for $n_{j} \leq n_{i}$, denotes the binomial coefficient and

$$
E\left\{\dot{\zeta}_{l}^{n}(t)\right\}=\frac{\Gamma\left(m_{l}+n / 2\right)}{\Gamma\left(m_{l}\right)}\left(\frac{\dot{\Omega}_{l}}{m_{l}}\right)^{n / 2}, \quad l=1,2, \ldots, L .
$$

By using this approximation for the PDF of a sum $\sum_{l=1}^{L} \zeta_{l}(t)$ of Nakagami- $m$ processes and applying the concept of transformation of random variables [[40], Equations 7-8], the PDF $p_{\Psi}(z)$ of the squared sum of Nakagami- $m$ processes $\Psi(t)$ can be expressed using $p_{\Psi}(z)=1 /(2 \sqrt{z}) p_{S}(\sqrt{z})$ as

$$
p_{\Psi}(z) \approx \frac{m_{S}^{m_{S}} z^{m_{S}-1}}{\Gamma\left(m_{S}\right) \Omega_{S}^{m_{S}}} \mathrm{e}^{-\frac{m_{S} \mathcal{Z}}{\Omega_{S}}}, \quad z \geq 0 .
$$

The joint PDF $p_{\Psi \dot{\Psi}}(z, \dot{z})$ can now be expressed with the help of [[16], Equation 19], (10) and by using the concept of transformation or random variables [[40], Equations 7-8] as

$$
p_{\Psi \dot{\Psi}}(z, \dot{z}) \approx \frac{\mathrm{e}^{-\frac{\dot{z}^{2}}{2 \sigma_{\dot{\psi}}^{2}}}}{\sqrt{2 \pi \sigma_{\dot{\Psi}}^{2}}} p_{\Psi}(z), \quad z \geq 0,|\dot{z}|<\infty
$$

where $\sigma_{\dot{\Psi}}^{2}=4 z\left(\pi f_{\max }\right)^{2} \sum_{l=1}^{L}\left(\dot{\Omega}_{l} / m_{l}\right)$. Using (10) and (11), the statistical properties of the capacity of Nakagami$m$ channels with EGC will be obtained in the next section.

\section{Statistical properties of the capacity of spatially correlated Nakagami- $m$ channels with diversity combining}

The instantaneous channel capacity $C(t)$ for the case when diversity combining is employed at the receiver can be expressed as [41]

$$
C(t)=\log _{2}(1+\gamma(t)) \quad(\text { bits } / \mathrm{s} / \mathrm{Hz})
$$

where $\gamma(t)$ represents the instantaneous SNR given by (3) and (7) for MRC and EGC, respectively. It is important to note that the instantaneous channel capacity $C(t)$ defined in (12) cannot always be reached by any proper coding schemes, since the design of coding schemes is based on the mean channel capacity (or the ergodic capacity). Nevertheless, it has been demonstrated in [29] that a study of the temporal behavior of the channel capacity can be useful in designing a system that can adapt the transmission rate according to the capacity evolving process in order to improve the overall system performance. The channel capacity $C(t)$ is a time-varying process and evolves in time as a random process. The expression in (12) can be considered as a mapping of the random process $\gamma(t)$ to another random process $C$ $(t)$. Hence, the statistical properties of the instantaneous SNR $\gamma(t)$ can be used to find the statistical properties of the channel capacity.

\subsection{Statistical properties of the capacity of spatially correlated Nakagami- $m$ channels with MRC}

The PDF $p_{\gamma}(z)$ of the instantaneous SNR $\gamma(t)$ can be found with the help of (4) and by employing the relation $p_{\gamma}(z)=\left(1 / \gamma_{s}\right) p_{\Xi}\left(z / \gamma_{s}\right)$. Thereafter, applying the concept of transformation of random variables, the PDF $p_{C}(r)$ of the channel capacity $C(t)$ is obtained using $p_{C}(r)=2^{r} \ln$ (2) $p_{\gamma}\left(2^{r}-1\right)$ as follows

$$
\begin{array}{r}
p_{C}(r)=\sum_{k=0}^{\infty} \frac{2^{r} \ln (2) \varepsilon_{k}\left(2^{r}-1\right)^{\sum_{l=1}^{L} \alpha_{l}+k-1} e^{-\frac{2^{r}-1}{\dot{\beta}_{1} \gamma_{s}}}}{\left(\dot{\beta}_{1} \gamma_{s}\right)^{\sum_{l=1}^{L} \alpha_{l}+k} \Gamma\left(\sum_{l=1}^{L} \alpha_{l}+k\right)} \\
\prod_{l=1}^{L}\left(\frac{\dot{\beta}_{1}}{\dot{\beta}_{l}}\right)^{\alpha_{l}}, \quad r \geq 0 .
\end{array}
$$

The CDF $F_{C}(r)$ of the channel capacity $C(t)$ can be found using the relationship $F_{C}(r)=\int_{0}^{r} p_{C}(x) d x$ [40]. After solving the integral, the CDF $F_{C}(r)$ of $C(t)$ can be expressed as

$$
F_{C}(r)=1-\prod_{l=1}^{L}\left(\frac{\dot{\beta}_{1}}{\dot{\beta}_{l}}\right)^{\alpha_{l}} \sum_{k=0}^{\infty} \frac{\varepsilon_{k} \Gamma\left(\sum_{l=1}^{L} \alpha_{l}+k, \frac{\left(2^{r}-1\right)}{\dot{\beta}_{1} \gamma_{\mathrm{s}}}\right)}{\Gamma\left(\sum_{l=1}^{L} \alpha_{l}+k\right)}
$$

for $r \geq 0$, where $\Gamma(\cdot, \cdot)$ represents the incomplete gamma function [[35], Equation 8.350-2].

In order to find the LCR $N_{C}(r)$ of the channel capacity $C(t)$, we first need to find the joint PDF $p_{C \dot{C}}(z, \dot{z})$ of the channel capacity $C(t)$ and its time derivative $\dot{C}(t)$. The joint PDF $p_{C \dot{C}}(z, \dot{z})$ can be obtained using 
$p_{\gamma \dot{\gamma}}(z, \dot{z})=\left(1 / \gamma_{s}^{2}\right) p_{\Xi \dot{\Xi}}\left(z / \gamma_{s}, \dot{z} / \gamma_{s}\right)$

where

$p_{\gamma \dot{\gamma}}(z, \dot{z})=\left(1 / \gamma_{s}^{2}\right) p_{\Xi \dot{\Xi}}\left(z / \gamma_{s}, \dot{z} / \gamma_{s}\right)$. The expression for the joint PDF $p_{C \dot{C}}(z, \dot{z})$ can be written as

$$
p_{C \dot{C}}(z, \dot{z})=\frac{2^{z} \ln (2) /\left(\pi f_{\max }\right)}{\sqrt{\left(2^{z}-1\right) 8 \pi \beta_{x} \gamma_{s}}} e^{-\frac{\left(2^{z} \ln (2) \dot{z}\right)^{2}}{8 \gamma_{s} \beta_{x}\left(2^{z}-1\right)\left(\pi f_{\max }\right)^{2}}} p_{C}(z)
$$

for $z \geq 0$ and $|\dot{z}|<\infty$. The LCR $N_{C}(r)$ can now be obtained by solving the integral in $N_{C}(r)=\int_{0}^{\infty} \dot{z} p_{C \dot{C}}(r, \dot{z}) d \dot{z}$. After some algebraic manipulations, the LCR $N_{C}(r)$ can finally be expressed in closed form as

$$
N_{C}(r)=\sqrt{\frac{2 \pi \beta_{x} \gamma_{s}\left(2^{r}-1\right)}{2^{2 r}\left(\ln (2) / f_{\max }\right)^{2}}} p_{C}(r), \quad r \geq 0 .
$$

The ADF $T_{C}(r)$ of the channel capacity $C(t)$ can be obtained using $T_{C}(r)=F_{C}(r) / N_{C}(r)$ [31], where $F_{C}(r)$ and $N_{C}(r)$ are given by (14) and (16), respectively.

\subsection{Statistical Properties of the Capacity of Spatially Correlated Nakagami- $m$ Channels with EGC}

For the case of EGC, the PDF $p_{\gamma}(z)$ of the instantaneous SNR $\gamma(t)$ can be obtained by substituting (10) in $p_{\gamma}(z)=\left(1 / \gamma_{s}\right) p_{\Psi}\left(z / \gamma_{s}\right)$, where $\dot{\gamma}_{s}=\gamma_{s} / L$. Thereafter, the PDF $p_{C}(r)$ is obtained by applying the concept of transformation of random variables on (7) as

$$
\begin{aligned}
p_{C}(r) & =2^{r} \ln (2) p_{\gamma}\left(2^{r}-1\right) \\
& \approx \frac{2^{r} \ln (2)\left(2^{r}-1\right)^{m_{S}-1}}{\Gamma\left(m_{S}\right)\left(\dot{\gamma}_{S} \Omega_{S} / m_{S}\right)^{m_{S}}} e^{-\frac{m_{S}\left(2^{r}-1\right)}{\hat{\gamma}_{s} \Omega_{S}}}, \quad r \geq 0 .
\end{aligned}
$$

By integrating the PDF $p_{C}(r)$, the CDF $F_{C}(r)$ of the channel capacity $C(t)$ can be obtained using $F_{C}(r)=\int_{0}^{r} p_{C}(x) d x$ as

$$
F_{C}(r) \approx 1-\frac{1}{\Gamma\left(m_{S}\right)} \Gamma\left(m_{S}, \frac{m_{S}\left(2^{r}-1\right)}{\gamma_{S} \Omega_{S}}\right), \quad r \geq 0
$$

The joint PDF $p_{C \dot{C}}(z, \dot{z})$, for the case of EGC, can be obtained

$$
\begin{aligned}
& p_{C \dot{C}}(z, \dot{z})=\left(2^{z} \ln (2)\right)^{2} p_{\gamma_{\dot{\gamma}}}\left(2^{z}-1,2^{z} \dot{z} \ln (2)\right) \\
& p_{\gamma \dot{\gamma}}(z, \dot{z})=\left(1 / \dot{\gamma}_{s}^{2}\right) p_{\Psi \dot{\Psi}}\left(z / \dot{\gamma}_{s}, \dot{z} / \dot{\gamma}_{s}\right) \text { as } \\
& p_{C \dot{C}}(z, \dot{z}) \approx \frac{e^{-\frac{\left(2^{z} \ln (2) \dot{z} /\left(\pi f_{\max }\right)\right)^{2}}{8 \dot{\gamma}_{s}\left(2^{z}-1\right)\left(\sum_{l=1}^{L} \hat{\Omega}_{l} / m_{l}\right)}} 2^{z} \ln (2) / f_{\max }}{\sqrt{\left(2^{z}-1\right) 8 \pi^{3}\left(\sum_{l=1}^{L} \Omega_{l} / m_{l}\right) \dot{\gamma}_{s}}} p_{C}(z)(19)
\end{aligned}
$$$$
\text { using }
$$

for $z \geq 0$ and $|\dot{z}|<\infty$. Now by employing the formula $N_{C}(r)=\int_{0}^{\infty} \dot{z} p_{C \dot{C}}(r, \dot{z}) d \dot{z}$, the LCR $N_{C}(r)$ of the channel capacity $C(t)$ can be approximated in closed form as

$$
N_{C}(r) \approx \sqrt{\frac{2 \pi\left(\sum_{l=1}^{L} \dot{\Omega}_{l} / m_{l}\right) \dot{\gamma}_{s}\left(2^{r}-1\right)}{2^{2 r}\left(\ln (2) / f_{\max }\right)^{2}}} p_{C}(r)
$$

for $r \geq 0$. By using $T_{C}(r)=F_{C}(r) / N_{C}(r)$, the ADF $T_{C}(r)$ of the channel capacity $C(t)$ can be obtained, while $F_{C}(r)$ and $N_{C}(r)$ are given by (18) and (20), respectively. It is noteworthy that although (17)-(20) represent approximate solutions, the numerical illustrations in the next section show no obvious deviation between these highly accurate approximations and the exact simulation results.

\section{Numerical results}

This section aims to analyze and to illustrate the analytical findings of the previous sections. The correctness of the analytical results will be confirmed with the help of exact simulations. For comparison purposes, we have shown the results for the mean channel capacity and the variance of the capacity of spatially correlated Rayleigh channels with MRC and EGC (obtained when $m_{l}=$ $1, \forall l=1,2, \ldots, L)$. Moreover, we have also presented the results for classical Nakagami- $m$ channels, which arise when $L=1$. In order to generate Nakagami- $m$ processes $\zeta_{l}(t)$, we have used the following relation [15]

$$
\zeta_{l}(t)=\sqrt{\sum_{i=1}^{2 \times m_{l}} \mu_{i, l}^{2}(t)}
$$

where $\mu_{i, l}(t)\left(i=1,2, \ldots, 2 m_{l}\right)$ are the underlying independent and identically distributed (i.i.d.) Gaussian processes, and $m_{l}$ is the parameter of the Nakagami- $m$ distribution associated with the $l$ th diversity branch. The Gaussian processes $\mu_{i, l}(t)$, each with zero mean and variances $\sigma_{0}^{2}$, were generated using the sum-of-sinusoids method [42]. The model parameters were calculated using the generalized method of exact Doppler spread $\left(\mathrm{GMEDS}_{1}\right)$ [43]. The number of sinusoids for the generation of the Gaussian processes $\mu_{i, l}(t)$ was chosen to be $N=20$. The SNR $\gamma_{s}$ was set to $15 \mathrm{~dB}$, the parameter $\Omega_{l}$ was assumed to be equal to $2 m_{l} \sigma_{0}^{2}$, the maximum Doppler frequency $f_{\max }$ was $91 \mathrm{~Hz}$, and the parameter $\sigma_{0}^{2}$ was equal to unity. Finally, using (21), (3), (7), and (12), the simulation results for the statistical properties of the capacity $C(t)$ of Nakagami- $m$ channels with MRC and EGC were obtained.

Figures 2 and 3 present the PDF $p_{C}(r)$ of the capacity of correlated Nakagami- $m$ channels with MRC and EGC, respectively, for different values of the number of diversity branches $L$ and receiver antennas separation $\delta_{R}$. It is observed that in both MRC and EGC, an 


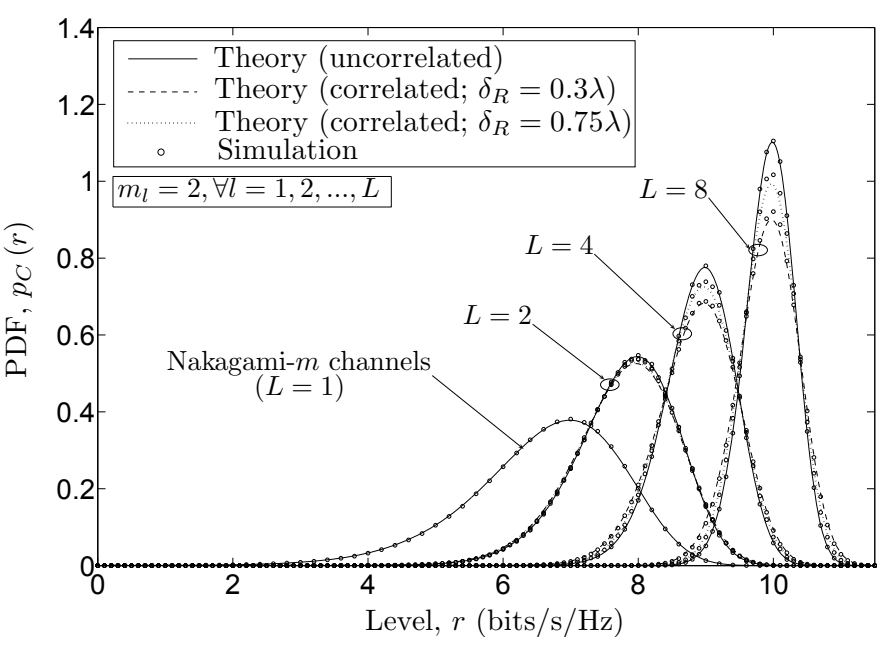

Figure 2 The PDF $p_{C}(r)$ of the capacity of correlated Nakagami- $m$ channels with MRC.

increase in the number of diversity branches $L$ increases the mean channel capacity. However, the variance of the channel capacity decreases. This fact is specifically highlighted in Figures 4 and 5, where the mean channel capacity and the variance of the capacity, respectively, of correlated Nakagami- $m$ channels is studied for different values of the number of diversity branches $L$ and receiver antennas separation $\delta_{R}$. The exact closed-form expressions for the mean $E\{C(t)\}$ and variance $\operatorname{Var}\{C(t)\}$ of the channel capacity cannot be obtained. Therefore, the results in Figures 4 and 5 are obtained numerically, using (17) and (13). It can be observed that the mean channel capacity and the variance of the capacity of Nakagami- $m$ channels are quite different from those of Rayleigh channels. Specifically, for both MRC and EGC, if the branches are less severely faded $\left(m_{l}=2, \forall l=1,2\right.$, ..., $L)$ as compared to Rayleigh fading $\left(m_{l}=1, \forall l=1,2\right.$,
..., $L$ ), then the mean channel capacity increases, while the variance of the channel capacity decreases.

The influence of spatial correlation on the PDF of the channel capacity is also studied in Figures 2 and 3. The results show that for Nakagami- $m$ channels with MRC, an increase in the spatial correlation in the diversity branches increases the variance of the channel capacity, while the mean channel capacity is almost unaffected. However, for the case of EGC, an increase in the spatial correlation decreases the mean channel capacity and has a minor influence on the variance of the channel capacity. Figures 4 and 5 also illustrate the effect of spatial correlation on the mean channel capacity and variance of the channel capacity, respectively, of Nakagami- $m$ channels with MRC and EGC. For the sake of completeness, we have also presented the results for the CDF of the capacity of correlated Nakagami- $m$ channels with

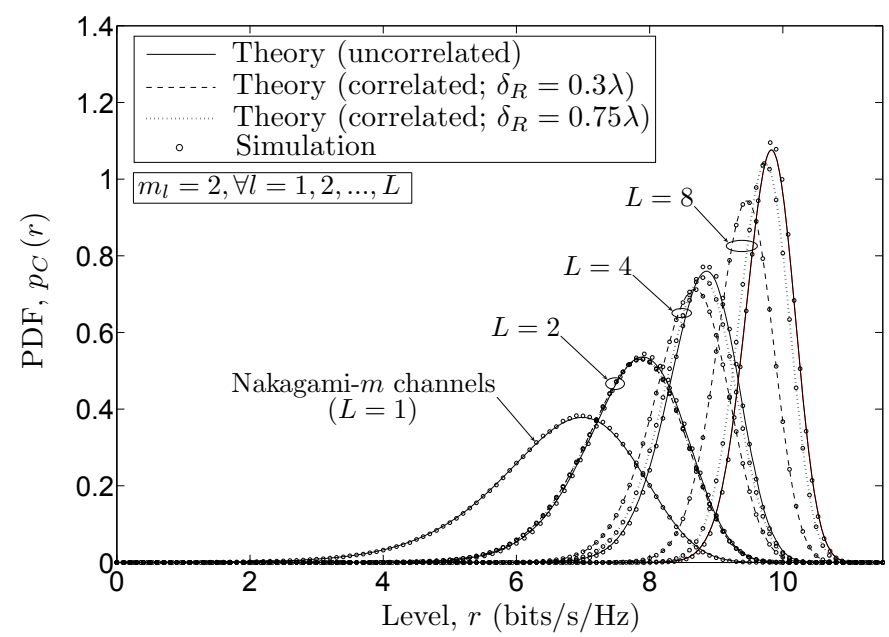

Figure 3 The PDF $p_{C}(r)$ of the capacity of correlated Nakagami- $m$ channels with EGC. 


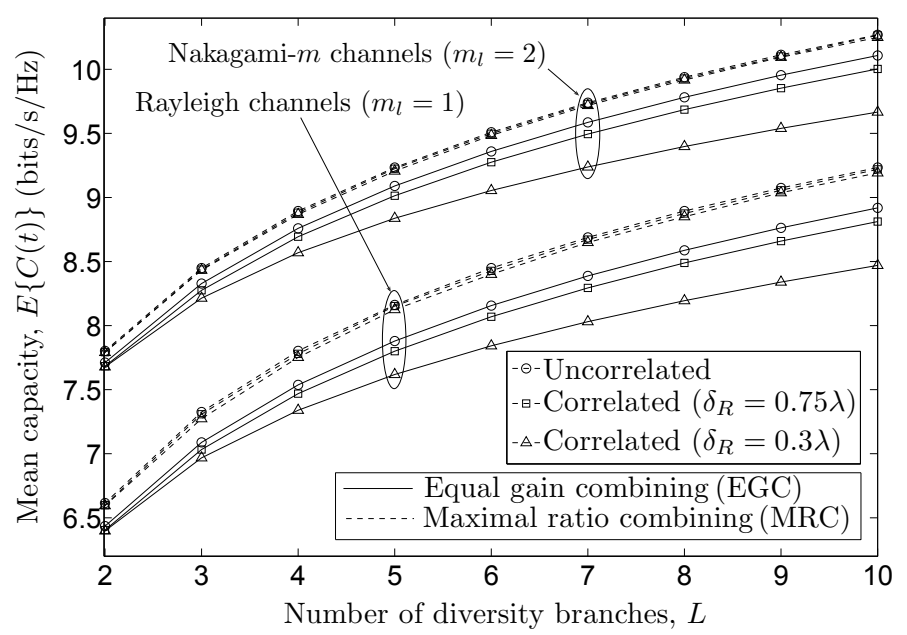

Figure 4 Comparison of the mean channel capacity of correlated Nakagami- $m$ channels with MRC and EGC.

MRC and EGC in Figures 6 and 7, respectively. Figures 6 and 7 can be studied to draw similar conclusions regarding the influence of the number of diversity branches $L$ as well as the spatial correlation on the mean channel capacity and the variance of the channel capacity as from Figures 2 and 3.

The LCR $N_{C}(r)$ of the capacity of Nakagami- $m$ channels with MRC and EGC is shown in Figures 8 and 9 for different values of the number of diversity branches $L$ and receiver antennas separation $\delta_{R}$. It can be seen in these two figures that at lower levels $r$, the LCR $N_{C}(r)$ of the capacity of Nakagami- $m$ channels with smaller values of the number of diversity branches $L$ is higher as compared to that of the channels with larger values of $L$. However, the converse statement is true for higher levels $r$. Moreover, an increase in the spatial correlation increases the LCR of the capacity of Nakagami- $m$ channels with MRC. On the other hand, when EGC is employed, an increase in the spatial correlation increases the LCR of the capacity of Nakagami- $m$ channels at only lower levels $r$, while the LCR decreases at the higher levels $r$.

The ADF $T_{C}(r)$ of the capacity of Nakagami- $m$ channels with MRC and EGC is studied in Figures 10 and 11 , respectively. The results show that the ADF of the capacity of Nakagami- $m$ channels with MRC decreases with an increase in the spatial correlation in the diversity branches. However, this effect is more prominent at higher levels $r$. On the other hand, when EGC is used, an increase in the spatial correlation increases the ADF of the channel capacity. Moreover for both MRC and EGC, an increase in the number of diversity branches decreases the ADF of the channel capacity. The analytical expressions are verified using simulations, whereby a very good fitting is found.

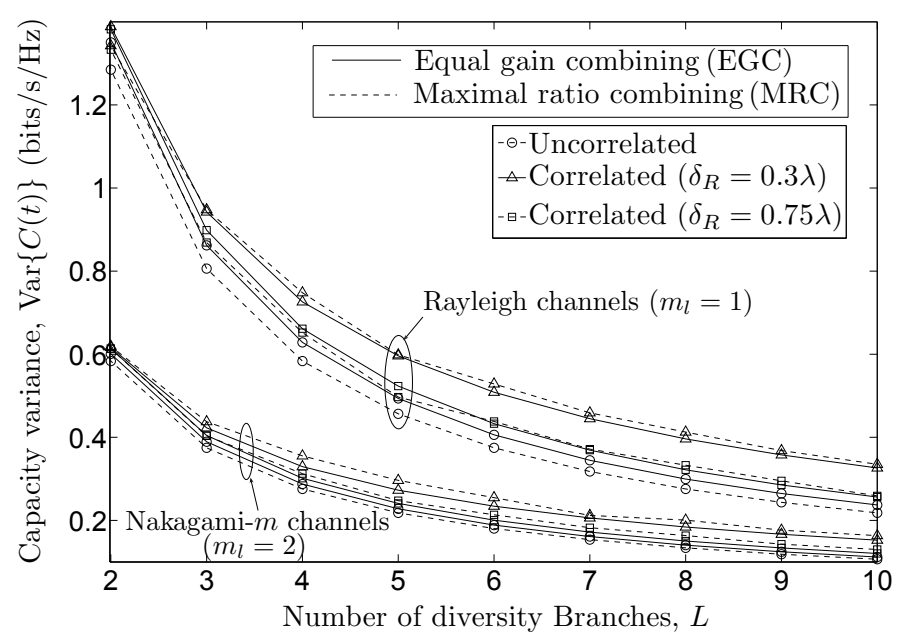

Figure 5 Comparison of the variance of the channel capacity of correlated Nakagami- $m$ channels with MRC and EGC. 


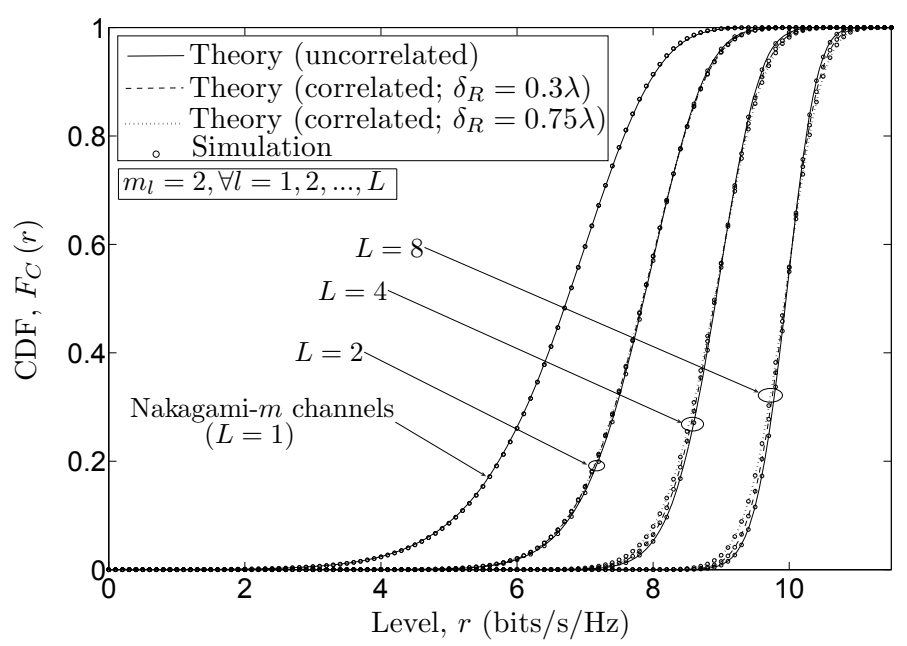

Figure 6 The CDF $F_{C}(r)$ of the capacity of correlated Nakagami- $m$ channels with MRC.

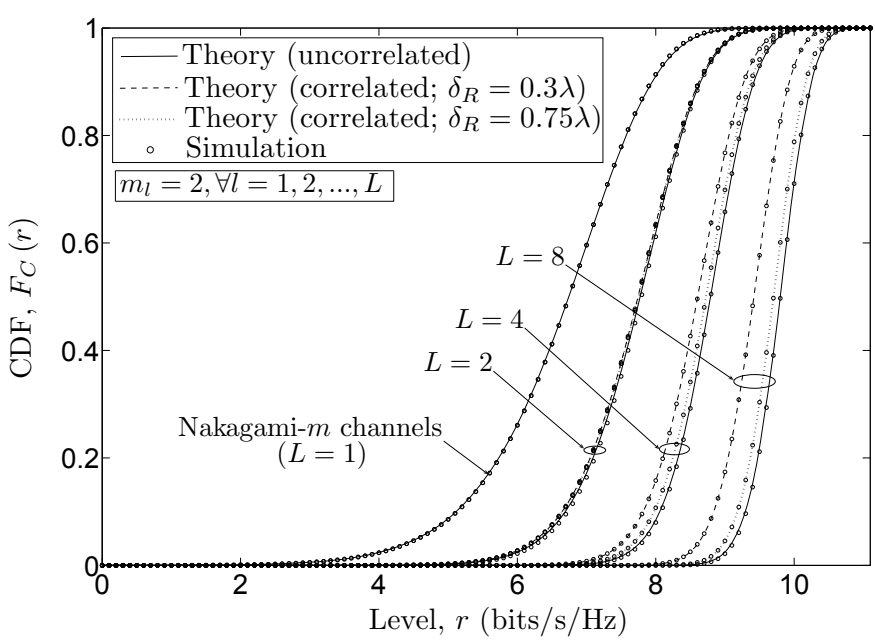

Figure 7 The CDF $F_{C}(r)$ of the capacity of correlated Nakagami- $m$ channels with EGC.

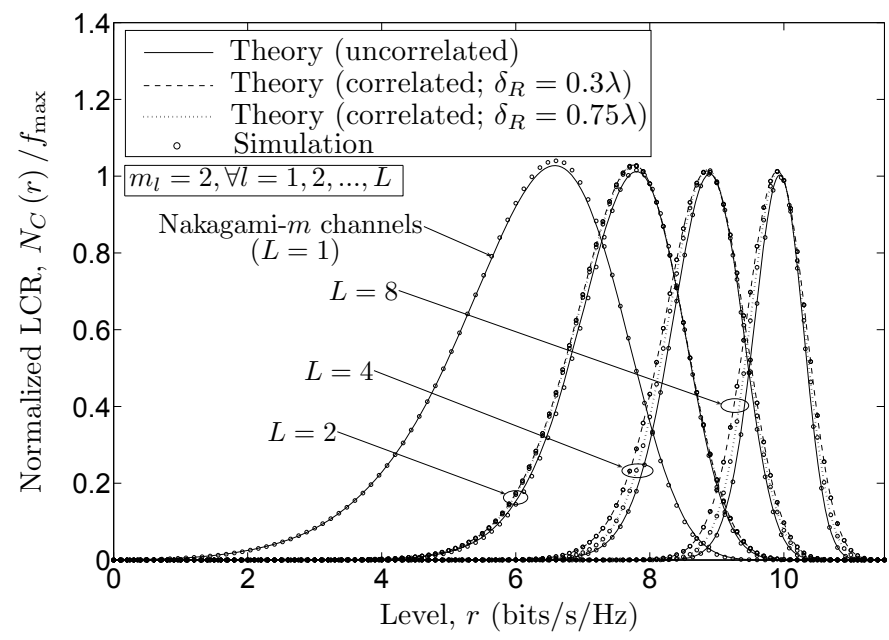

Figure 8 The normalized LCR $N_{C}(r) / f_{\max }$ of the capacity of correlated Nakagami- $m$ channels with MRC 


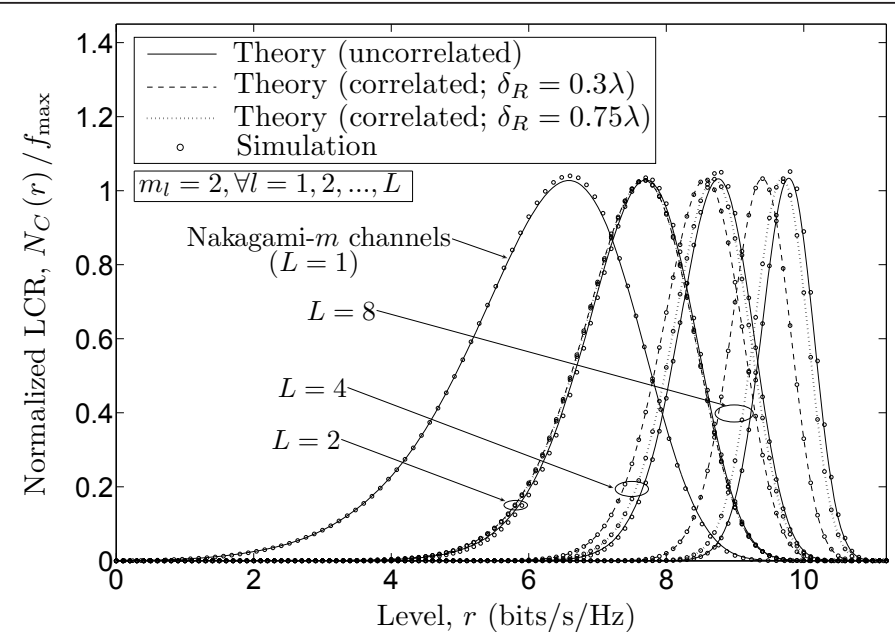

Figure 9 The normalized LCR $N_{C}(r) / f_{\max }$ of the capacity of correlated Nakagami- $m$ channels with EGC.

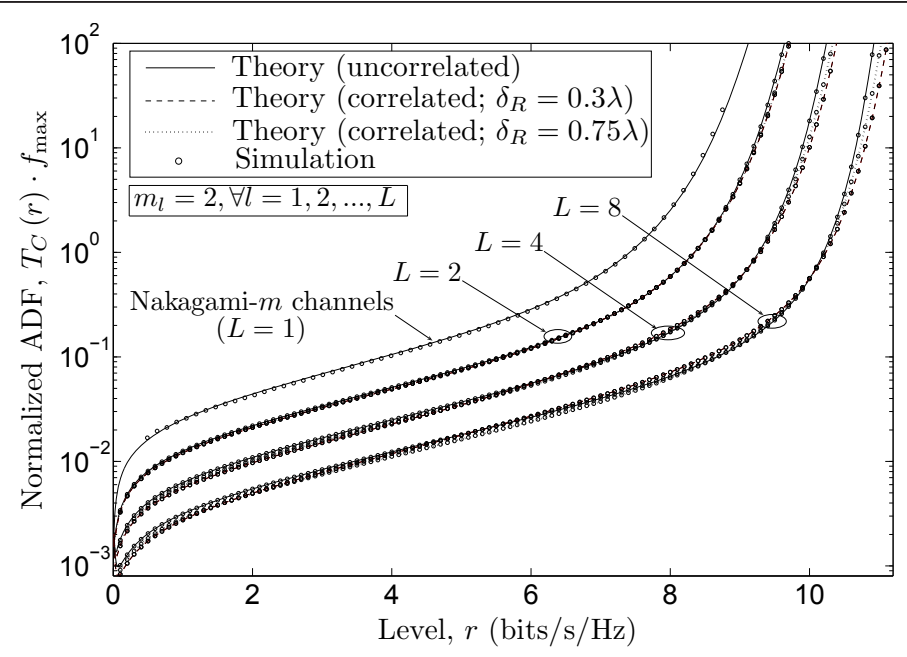

Figure 10 The normalized ADF $T_{C}(r) \cdot f_{\max }$ of the capacity of correlated Nakagami- $m$ channels with MRC.

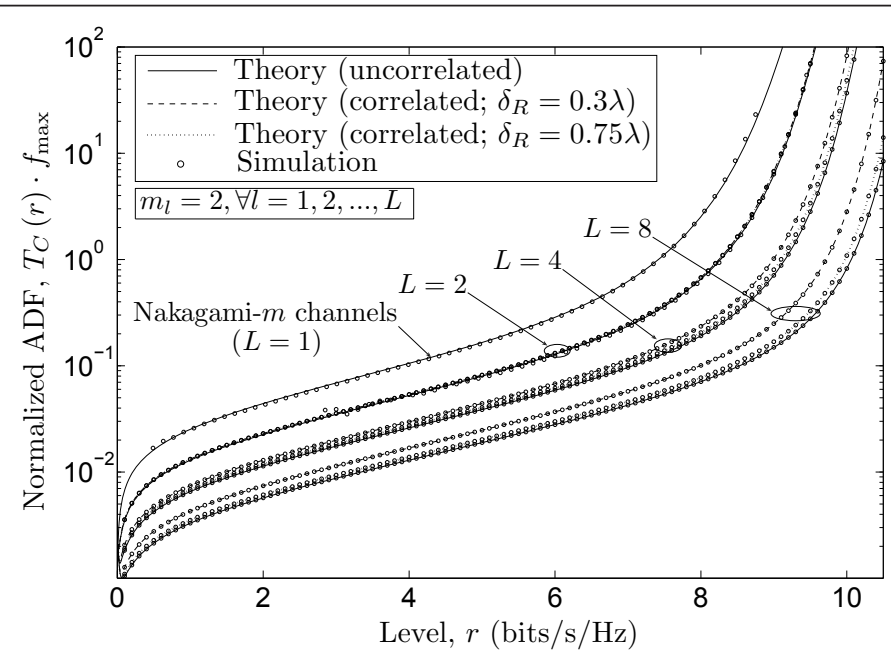

Figure 11 The normalized ADF $T_{C}(r) \cdot f_{\max }$ of the capacity of correlated Nakagami- $m$ channels with EGC. 


\section{Conclusion}

This article studies the statistical properties of the capacity of spatially correlated Nakagami- $m$ channels with MRC and EGC. We have derived analytical expressions for the PDF, CDF, LCR, and ADF of the capacity of Nakagami- $m$ channels with MRC and EGC. The results are studied for different values of the number of diversity branches $L$ and receiver antennas separation $\delta_{R}$. It is observed that for MRC, an increase in the spatial correlation increases the variance as well as the LCR of the channel capacity; however, the ADF of the channel capacity decreases. On the other hand, when using EGC, an increase in the spatial correlation decreases the mean channel capacity, whereas the ADF of the channel capacity increases. Moreover, an increase in the spatial correlation increases the LCR of the channel capacity at only lower levels $r$. It is also observed that for both MRC and EGC, an increase in the number of diversity branches increases the mean channel capacity, while the variance and ADF of the channel capacity decrease. The results also show that at lower levels, the LCR is higher for channels with smaller values of the number of diversity branches $L$ than for channels with larger values of $L$. The analytical findings are verified using simulations, where a very good agreement between the theoretical and simulation results was observed.

\section{Endnotes}

${ }^{a}$ By instantaneous channel capacity we mean the timevariant channel capacity $[44,45]$. In the literature, it is also known as the maximum mutual information [46-48].

${ }^{\mathrm{b}}$ The scope of this paper is limited only to the derivation and analysis of the statistical properties of the instantaneous channel capacity. However, a detailed discussion on this topic can be found in, e.g., $[29,49,50]$ and the references therein.

${ }^{\mathrm{c}}$ Henceforth, for ease of notation, we will call the instantaneous channel capacity as simply the channel capacity (similar notation is also used in $[34,41,51]$ ).

\section{Author details \\ ${ }^{1}$ Faculty of Engineering and Science, University of Agder, P.O.Box 509, NO- 4898 Grimstad, Norway ${ }^{2}$ Centro de Investigación y, de Estudios Avanzados, CINVESTAV, Mexico City, Mexico}

\section{Competing interests}

The authors declare that they have no competing interests.

Received: 3 March 2011 Accepted: 30 September 2011 Published: 30 September 2011

\section{References}

1. WC Jakes (ed.), Microwave Mobile Communications, (IEEE Press, Piscataway, NJ, 1994)
2. WCY Lee, Mobile Communications Engineering, 2nd edn., (McGraw-Hill, New York, 1998)

3. J Salz, JH Winters, Effect of fading correlation on adaptive arrays in digital mobile radio. IEEE Trans Veh Technol. 43(4), 1049-1057 (1994). doi:10.1109/ 25.330168

4. EA Jorswieck, TJ Oechtering, H Boche, Performance analysis of combining techniques with correlated diversity, in Proceedings of IEEE Wireless Communications and Networking Conference, WCNC 2005, 2, 849-854 (2005)

5. P Lombardo, G Fedele, MM Rao, MRC performance for binary signals in Nakagami fading with general branch correlation. IEEE Trans Commun. 47(1), 44-52 (1999). doi:10.1109/26.747812

6. Y Chen, C Tellambura, Performance analysis of L-branch equal gain combiners in equally correlated Rayleigh fading channels. IEEE Commun Lett. 8(3), 150-152 (2004). doi:10.1109/LCOMM.2004.825722

7. WR Young, Comparison of mobile radio transmission at 150,450, 900, and $3700 \mathrm{MHz}$. Bell Syst Technol J. 31, 1068-1085 (1952)

8. SO Rice, Mathematical analysis of random noise. Bell Syst Tech J. 24 46-156 (1945)

9. DM Black, DO Reudink, Some characteristics of mobile radio propagation at $836 \mathrm{MHz}$ in the Philadelphia area. IEEE Trans Veh Technol. 21, 45-51 (1972)

10. DO Reudink, Comparison of radio transmission at X-band frequencies in suburban and urban areas. IEEE Trans Antenna Propag. 20(4), 470-473 (1972). doi:10.1109/TAP.1972.1140240

11. M-S Alouini, AJ Goldsmith, Capacity of Rayleigh fading channels under different adaptive transmission and diversity-combining techniques. IEEE Trans Veh Technol. 48(4), 1165-1181 (1999). doi:10.1109/25.775366

12. S Khatalin, JP Fonseka, On the channel capacity in Rician and Hoyt fading environments with MRC diversity. IEEE Trans Veh Technol. 55(1), 137-141 (2006). doi:10.1109/TVT.2005.861205

13. M Nakagami, The $m$-distribution: a general formula of intensity distribution of rapid fading, in Statistical Methods in Radio Wave Propagation, ed. by Hoffman WG (Pergamon Press, Oxford, UK, 1960)

14. SH Choi, P Smith, B Allen, WQ Malik, M Shafi, Severely fading MIMO channels: models and mutual information, in Proceedings of IEEE International Conference on Communications, ICC 2007, Glasgow, UK, 4628-4633 (2007)

15. MD Yacoub, JEV Bautista, LG de Rezende Guedes, On higher order statistics of the Nakagami-m distribution. IEEE Trans Veh Technol. 48(3), 790-794 (1999)

16. MD Yacoub, CRCM da Silva, JEV Bautista, Second-order statistics for diversity-combining techniques in Nakagami-fading channels. IEEE Trans Veh Technol. 50(6), 1464-1470 (2001). doi:10.1109/25.966577

17. G Rafiq, V Kontorovich, M Pätzold, The influence of severity of fading on the statistical properties of the capacity of Nakagami-m channels with MRC and EGC, in Proceedings of 2010 European Wireless Conference, EW 2010, 406-410 (2010)

18. H Samimi, P Azmi, An approximate analytical framework for performance analysis of equal gain combining technique over independent Nakagami, Rician and Weibull fading channels. Wireless Pers Commun. 43(4), 1399-1408 (2007). doi:10.1007/s11277-007-9314-z

19. VA Aalo, Performance of maximal-ratio diversity systems in a correlated Nakagami-fading environment. IEEE Trans Commun. 43(8), 2360-2369 (1995). doi:10.1109/26.403769

20. QT Zhang, Exact analysis of postdetection combining for DPSK and NFSK systems over arbitrarily correlated Nakagami channels. IEEE Trans Commun. 46(11), 1459-1467 (1998). doi:10.1109/26.729390

21. QT Zhang, Maximal-ratio combining over Nakagami fading channels with an arbitrary branch covariance matrix. IEEE Trans Veh Technol. 48(4), 1141-1150 (1999). doi:10.1109/25.775363

22. C Mun, C Kang, H Park, Approximation of SNR statistics for MRC diversity systems in arbitrarily correlated Nakagami fading channels. 35(4), 266-267 (1999)

23. $M Z$ Win, JH Winters, Exact error probability expressions for MRC in correlated Nakagami channels with unequal fading parameters and branch powers, in Proceedings of IEEE Global Telecommunications Conference, GLOBECOM 1999, 5, 2331-2335 (1999)

24. S Khatalin, JP Fonseka, Capacity of correlated Nakagami- $m$ fading channels with diversity combining techniques. IEEE Trans Veh Technol. 55(1), 142-150 (2006). doi:10.1109/TVT.2005.861206

25. G Rafiq, V Kontorovich, M Pätzold, On the statistical properties of the capacity of the spatially correlated Nakagami-m MIMO channels, in 
Proceedings of IEEE 67th Vehicular Technology Conference, IEEE VTC 2008Spring, Marina Bay, Singapore, 500-506 (2008)

26. J Proakis, M Salehi, Digital Communications, 5th edn. (McGraw-Hill, New York, 2008)

27. D Gesbert, J Akhtar, Breaking the barriers of Shannon's capacity: an overview of MIMO wireless systems. Telenor's Journal Telektronikk: Information theory and its applications 1.2002, 53-64 (2002)

28. AJ Paulraj, RU Nabar, DA Gore, Introduction to Space-Time Wireless Communications, (Cambridge University Press, Cambridge, UK, 2003)

29. M Luccini, A Shami, S Primak, Cross-layer optimization of network performance over multiple-input multiple-output wireless mobile channels. 4(6), 683-696 (2010)

30. BO Hogstad, M Pätzold, Capacity studies of MIMO models based on the geometrical one-ring scattering model, in Proceedings of 15th IEEE International Symposium on Personal, Indoor and Mobile Radio Communications, PIMRC 2004, vol. 3. Barcelona, Spain, pp. 1613-1617 (2004)

31. BO Hogstad, M Pätzold, Exact closed-form expressions for the distribution, level-crossing rate, and average duration of fades of the capacity of MIMO channels, in Proceedings of 65th Semiannual Vehicular Technology Conference, IEEE VTC 2007-Spring. Dublin, Ireland, 455-460 (2007)

32. K Otani, K Daikoku, H Omori, Burst error performance encountered in digital land mobile radio channel. IEEE Trans Veh Technol. 30(4), 156-160 (1981)

33. R Vijayan, JM Holtzman, Foundations for level crossing analysis of handoff algorithms, in Proceedings of IEEE International Conference on Communications, ICC 1993. Geneva, Switzerland, 935-939 (1993)

34. A Giorgetti, PJ Smith, M Shafi, M Chiani, MIMO capacity, level crossing rates and fades: the impact of spatial/temporal channel correlation. J Commun Netw. 5(2), 104-115 (2003)

35. IS Gradshteyn, IM Ryzhik, Table of Integrals, Series, and Products, 6th edn. (Academic Press, 2000)

36. GJ Byers, F Takawira, Spatially and temporally correlated MIMO channels: modelling and capacity analysis. IEEE Trans Veh Technol. 53(3), 634-643 (2004). doi:10.1109/TVT.2004.825766

37. M-S Alouini, A Abdi, M Kaveh, Sum of gamma variates and performance of wireless communication systems over Nakagami-fading channels. IEEE Trans Veh Technol. 50(6), 1471-1480 (2001). doi:10.1109/25.966578

38. A Annamalai, C Tellambura, VK Bhargava, Equal-gain diversity receiver performance in wireless channels. IEEE Trans Commun. 48(10), 1732-1745 (2000). doi:10.1109/26.871398

39. DB da Costa, MD Yacoub, JCS Santos Filho, An improved closed-form approximation to the sum of arbitrary Nakagami- $m$ variates. IEEE Trans Veh Technol. 57(6), 3854-3858 (2008)

40. A Papoulis, SU Pillai, Probability, Random Variables and Stochastic Processes, 4th edn. (McGraw-Hill, New York, 2002)

41. GJ Foschini, MJ Gans, On limits of wireless communications in a fading environment when using multiple antennas. Wireless Pers Commun. 6, 311-335 (1998). doi:10.1023/A:1008889222784

42. M Pätzold, Mobile Fading Channels (Wiley, Chichester, 2002)

43. M Pätzold, CX Wang, BO Hogstad, Two new sum-of-sinusoids-based methods for the efficient generation of multiple uncorrelated Rayleigh fading waveforms. IEEE Trans Wirel Commun. 8(6), 3122-3131 (2009)

44. G Fraidenraich, O Leveque, JM Cioffi, On the MIMO channel capacity for the Nakagami-m channel. IEEE Trans Inf Theory 54(8), 3752-3757 (2008)

45. N Costa, S Haykin, Multiple-Input, Multiple-Output Channel Models: Theory and Practice (Wiley, New Jersey, 2010)

46. IE Telatar, Capacity of multi-antenna Gaussian channels. Eur Trans Telecommun Relat Technol. 10, 585-595 (1999). doi:10.1002/ett.4460100604

47. A Tulino, A Lozano, S Verdu, Impact of antenna correlation on the capacity of multiantenna channels. IEEE Trans Inf Theory 51(7), 2491-2509 (2005). doi:10.1109/TIT.2005.850094

48. M Kang, SM Alouini, Capacity of MIMO Rician channels. IEEE Trans Wireless Commun. 5(1), 112-122 (2006)

49. AJ Goldsmith, SG Chua, Variable-rate variable-power MQAM for fading channels. IEEE Trans Commun. 45(10), 1218-1230 (1997). doi:10.1109/ 26.634685

50. AJ Goldsmith, P Varaiya, Capacity of fading channels with channel side information. IEEE Trans Inf Theory 43(6), 1986-1992 (1997). doi:10.1109/ 18.641562

51. PJ Smith, LM Garth, S Loyka, Exact capacity distributions for MIMO systems with small numbers of antennas. IEEE Commun Lett. 7(10), 481-483 (2003). doi:10.1109/LCOMM.2003.817318 doi:10.1186/1687-1499-2011-116

Cite this article as: Rafiq et al: The impact of spatial correlation on the statistical properties of the capacity of nakagami- $m$ channels with MRC and EGC. EURASIP Journal on Wireless Communications and Networking $20112011: 116$

\section{Submit your manuscript to a SpringerOpen ${ }^{\mathcal{O}}$ journal and benefit from:}

- Convenient online submission

- Rigorous peer review

- Immediate publication on acceptance

- Open access: articles freely available online

- High visibility within the field

- Retaining the copyright to your article

Submit your next manuscript at $\gg$ springeropen.com 